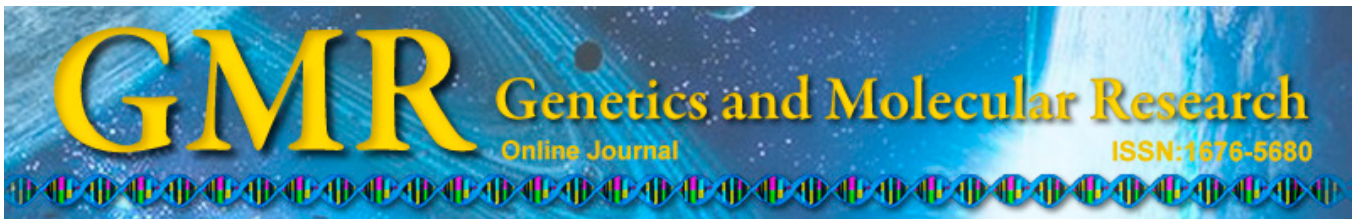

\title{
Development of an improved rat model of dual graft liver transplantation with long-term survival
}

\author{
X.-J. Xie ${ }^{1,2}$, Y.-F. Ye ${ }^{1,2}$, Y. He ${ }^{1,2}$, H.-Y. Xie ${ }^{1,2}$, L. Zhou ${ }^{1,2}$, Y. Wang ${ }^{1,2}$, \\ C.-Y. Xing ${ }^{1,2}$ and S.-S. Zheng ${ }^{1,2}$ \\ ${ }^{1}$ Key Laboratory of Combined Multi-Organ Transplantation, \\ Key Laboratory of Organ Transplantation, Ministry of Public Health, \\ Hangzhou Zhejiang, China \\ ${ }^{2}$ Division of Hepatobiliary and Pancreatic Surgery, Department of Surgery, \\ First Affiliated Hospital, Zhejiang University School of Medicine, \\ Zhejiang Province, China \\ Corresponding author: S.-S. Zheng \\ E-mail: shusenzhengcn@126.com
}

Genet. Mol. Res. 13 (3): 8035-8045 (2014)

Received May 23, 2014

Accepted September 11, 2014

Published September 29, 2014

DOI http://dx.doi.org/10.4238/2014.September.29.16

\begin{abstract}
Dual graft liver transplantation has been demonstrated to be feasible as well as effective in increasing the donor pool and in preventing the potential for small-for-size syndrome. However, little is known about the pathophysiological and immune processes following dual graft liver transplantation due to the lack of appropriate animal models. The aim of this study, therefore, was to establish an improved rat model of dual graft liver transplantation, with long-term survival. Male inbred rats were used as both donors and recipients. One middle lobe together with another right middle lobe from the livers of two different donors were used as the dual grafts. The "basinshaped anastomosis" technique was used to connect the suprahepatic inferior vena cava; "Y-shaped bridge" and "three-cuff" techniques were adopted for the anastomosis of the portal veins; and the "twostent" technique was used for the anastomosis of the bile ducts. Six
\end{abstract}


of the ten recipients survived for more than 100 days after dual graft liver transplantation. There was no difference in graft survival between dual and whole liver transplantation. The long-term survivors with dual grafts from two different donors had unobstructed portal vein flow, unobstructed biliary tract dilatation, normal graft function, and well-preserved hepatic structure. Therefore, this improved model will be potentially useful for evaluating the pathophysiological processes, immune responses between dual grafts and recipient, and mechanisms underlying the liver regeneration in dual grafts after dual graft liver transplantation.

Key words: Dual graft liver transplantation; Y-shaped bridge; Basin-shaped anastomosis; Small-for-size syndrome

\section{INTRODUCTION}

Liver transplantation has been widely accepted as the most effective therapy for endstage liver diseases. However, a severe shortage of cadaveric organ donors remains a major obstacle preventing the development of liver transplantation (Wertheim et al., 2011). Several innovative techniques such as split liver transplantation (SLT) and living donor liver transplantation (LDLT) have been developed to enlarge the relatively constant pool of organs (Strong et al., 1990; Hashikura et al., 1994; Yersiz et al., 2006). However, the inadequate liver graft volume in the recipient and remnant liver volume in the donor has always been a major issue in both SLT and LDLT, potentially leading to small-for-size syndrome (SFSS), which remains one of the principal factors restricting the progress of SLT and LDLT (Dahm et al., 2005). To prevent the incidence of SFSS following LDLT, the technique of dual graft liver transplantation (DLT) has been introduced, in which one recipient receives lobe grafts from each of two living donors (Lee et al., 2001a). DLT offers both a sufficient graft size for the adult recipient as well as a safe remnant liver volume for the donor. The first successful human DLT was reported by Lee et al. (2001a). To date, cases of DLT have been reported worldwide, especially in Asian countries such as Korea (Lee et al., 2001b), Japan (Soejima et al., 2008) and China (Chen et al., 2010). In our transplant center, we performed the first DLT in a patient with acute-on-chronic hepatitis B liver failure in 2010 (Zheng SS, Wu J, Xu X, Sheng Y, et al., unpublished result). Nowadays, DLT has been demonstrated to be feasible as well as effective in increasing the donor pool and in preventing the potential for SFSS (Chen et al., 2013). However, little is known about the pathophysiological and immune processes following DLT due to the lack of appropriate animal models. Although Zhang et al. (2012) reported the first rat model of DLT in 2012, the 7-day cumulative survival rate was only $58.3 \%$. Therefore, there is an urgent need to develop an animal model of DLT with long-term survival. Obstacles toward developing a reliable rat model of DLT with long-term survival include the difficulties encountered in anastomosis of the supra/infrahepatic inferior venae cavae (SHIVC and IHIVC, respectively), portal veins (PV) and bile ducts between the dual grafts and the recipient. This article presents an improved technique to augment the rat model of DLT with long-term survival (>100 days), which will facilitate further research into the pathophysiology and immunology of DLT. 


\section{MATERIAL AND METHODS}

\section{Animals}

Male inbred Sprague Dawley rats, 10 to 12 weeks old, weighing from 250 to 300 $\mathrm{g}$, were used as isograft transplant donors and recipients. The study was conducted in accordance with the Guidelines for the Care and Use of Laboratory Animals and was approved by the Animal Ethics Review Committees of Zhejiang University. All animals were kept in a temperature-controlled environment with a 12-h light-dark cycle; food and water were given ad libitum.

\section{Experimental design}

Syngeneic orthotopic whole liver transplantations (WLT) were performed according to Kamada and Calne (1979). An improved rat model of DLT was developed using grafts from each of two living donors: one median lobe together with a right median lobe, as described in detail below. The rats were followed daily until death or random sacrifice on postoperative days 7 and 100 for assessment of surgical techniques, and histopathologic examination.

\section{First donor operation}

A cross midline incision on the abdomen was made. The falciform and triangular ligaments were cut. The left inferior phrenic vein was ligated with thread. The caudate lobe was ligated with a 6-0 prolene suture and then resected. The common bile duct (CBD) was cut at the level of the pancreas. A biliary stent tube was inserted into the CBD towards the hepatic side (5 mm length, $1 \mathrm{~mm}$ outer diameter, and slant cuts at both ends) and fixed appropriately. A second biliary stent tube was inserted into the left hepatic duct. The left lobe, right upper lobe, and right lower lobe were transfixed with 6-0 prolene sutures and then resected, with only the middle lobe retained. The IHIVC was skeletonized. Heparin (100 U/rat) was administered intravenously through the IHIVC. After puncture of the end segment of the abdominal aorta, a thoracotomy was performed immediately and the thoracic aorta was clamped. The thoracic inferior vena cava and IHIVC were cut to start an outflow of lavage solution. Lavage was then performed through the aorta with $10 \mathrm{~mL}$ of Ringer's solution $\left(0^{\circ}\right.$ to $4^{\circ} \mathrm{C}$, including 12.5 $\mathrm{U}$ heparin) at the rate of $2.5 \mathrm{~mL} / \mathrm{min}$. The SHIVC was transected when the donor liver graft became pallid. The pyloric and splenic veins were ligated closed to the PV and the distal part of pyloric and splenic veins were then transected. The PV was cut off approximately $2 \mathrm{~mm}$ below the ligature of the splenic vein. Finally, the first donor liver graft was harvested and immediately placed into ice-cold Ringer's solution (Figure 1A).

\section{Second donor operation}

The second liver graft was harvested in a similar manner as the first. The main procedural differences were that the left middle lobe was also resected, leaving the right middle lobe for harvest, and the biliary stent tubes did not need to be inserted. The IHIVC was re- 
sected together with the left renal vein (each at $5 \mathrm{~mm}$ in length) and all branches of the veins (testicular vein, left adrenal vein and lumbar vein) were ligated beforehand. The left renal vein and the IHIVC were Y-shaped and could be used as the bypass vessels of the PV (Figure 1B).
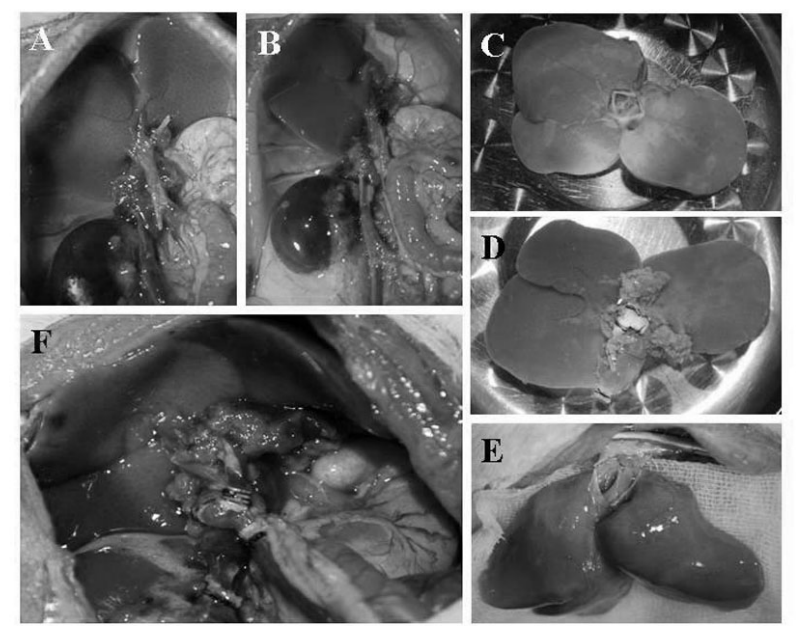

G

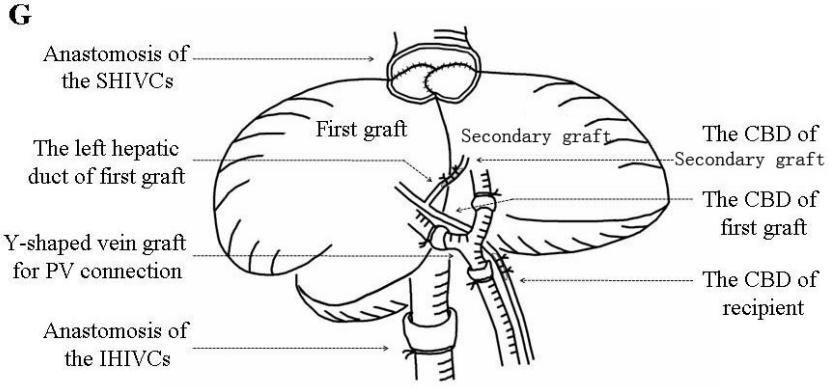

Figure 1. Dual graft liver transplantation (DLT) operation procedures. A. Middle liver lobe was used as the first graft. Two stents were prepared in the common bile duct and the left hepatic bile duct, respectively. B. Right middle liver lobe of another donor was used as the second graft. Intrahepatic inferior vena cava (IHIVC) and the left renal vein were dissected as the bypass vein for connecting the portal veins (PVs). C. Superhepatic inferior venae cavae (SHIVCs) from the two grafts were sutured into a discoid shape. D. PVs of the dual grafts were bridged using the prepared bypass vein. Common bile duct (CBD) of second graft was connected to the left hepatic bile duct of first graft. E. Dual grafts were implanted. The SHIVCs of the dual grafts were anastomosed to the SHIVC of recipient. F. Last branch of the bypass vein was sleeved into the recipient's PV. The IHIVC of the first graft was sleeved into the recipient's IHIVC. The stent of the first graft's CBD was inserted into the recipient's CBD. G. Anastomosis of the SHIVC, IHIVC, PV, and bile duct between the dual grafts and the recipient.

\section{Plasty of the liver grafts}

The procedure was performed on ice with Ringer's solution $\left(4^{\circ} \mathrm{C}\right)$. Three polyethylene plastic PV and one IHIVC cuffs were used. The inner and outer diameters of the PV cuffs were 1.8 and $2.1 \mathrm{~mm}$, while that of IHIVC cuff was 2.6 and $2.8 \mathrm{~mm}$, respectively. The diaphragmatic surfaces of the dual grafts were placed upward. The second liver graft was rotated through 
$180^{\circ}$ so that the dual liver grafts were pieced into a mirrored shape. The posterior walls of the SHIVCs from the dual grafts were continuously sutured with 8-0 prolene suture. The openings of the two SHIVCs from the dual liver grafts were sutured into a discoid shape (Figure 1C). The stent tube of the left hepatic duct of the first liver graft was inserted into the CBD of the second liver graft. The "Y-shaped bridge" method was adopted for the anastomosis of the PV. The bypass vessel was introduced through the PV cuffs. Two ends of the Y-shaped blood vessel were led into the PVs of the first and second grafts. Another cuff was prepared for the PV of the recipient (Figure 1D). After attachment of the cuffs to the PV and IHIVC, the patency of the cuffs and the branch closure were checked using a flush of Ringer's solution through a 24 gauge catheter. The IHIVC cuff of the first liver graft was also attached using a similar procedure. The IHIVC of the second donor was ligated and then affixed to the midpoint of the anterior wall of the SHIVC.

\section{Recipient operation}

The abdomen of the recipient was opened through a long midline incision. The procedures for removal of the native liver were largely the same as those for removal of the donor liver. The branches of the PV connecting to the paraesophageal vessels were ligated. The proper hepatic arteries were transfixed. The cut-off point of the CBD was close to the first hepatic portal. The IHIVC was blocked by a vascular clamp above the renal vein and the PV was blocked at the level of the pyloric vein. Anhepatic phase was initiated, followed by injection of 2.0 to $2.5 \mathrm{~mL}$ of lactated Ringer's solution into the liver by puncture at the crotch of the PV, until the liver became pallid. The recipient's liver was gently pulled down using a previously placed rubber strap. The SHIVC was clamped and cut off at the liver parenchyma. The PV was cut close to the hilum and the recipient's liver was then removed.

\section{Implantation of dual liver grafts}

The two liver grafts were shifted out from the ice bath and orthotopically imbedded into the right upper abdominal cavity. The first graft was positioned on the right side and the second graft on the left side. The anastomotic line of the donor SHIVCs was positioned perpendicular to the coronal axis of the recipient SHIVC. The stay sutures of the liver grafts were combined with the marginal part of the recipient SHIVC. The posterior wall was sutured using 10 to 15 stitches of continuous sutures (Figure 1E). The anterior wall was then sutured using 15 to 20 stitches of continuous sutures. When sewing to the midpoint, care was taken to ensure all knots were tied outside of the blood vessel to avoid thrombosis. The stitches were denser than those in WLT to avoid bleeding. An L-shaped injector was used to fill the SHIVC cavity with Ringer's solution to rinse off the gas bubbles before complete closure. The last branch of the bridge vein cannula was sleeved into the cavity of the recipient PV and fixed. The blockade clamps of the SHIVC and the PV were removed to restore hepatic blood supply. The first donor's IHIVC cannula was sleeved into the cavity of the recipient IHIVC. The clamp of the IHIVC was then removed to restore blood flow. The stent of the first donor's CBD was inserted into the recipient's bile duct and then ring ligated (Figure 1F). The anastomosis of the SHIVC, IHIVC, PVs, and bile ducts between the dual grafts and the recipient is shown in Figure 1G. Before abdominal closure, the intraperitoneal cavity was washed with warm saline 
solution, followed by administration of cefazolin $(100 \mathrm{mg} / \mathrm{kg})$ into the abdominal cavity to prevent infection. The abdominal incision was closed by whole layer continuous suture. The rats were kept on a warming blanket and under a heat lamp postoperatively until resuscitation. All recipients were allowed to spontaneously recover without further treatment (such as immunosuppression).

\section{Histology}

Complete necropsies were performed on all animals to determine the cause of death. Tissues from sacrificed animals were fixed in $10 \%(\mathrm{v} / \mathrm{v})$ neutral buffered formalin, embedded in paraffin, and sectioned. The 4- $\mu \mathrm{m}$ sections were stained with hematoxylin and eosin for histological examination.

\section{Magnetic resonance imaging (MRI)}

MRI was performed to assess the morphology and regeneration of the dual liver grafts after the operation.

\section{Digital subtraction angiography (DSA)}

DSA was performed to assess flow within the hepatic venous system and biliary tract after vascular reconstruction. Ten rats in the WLT and DLT groups were chosen for survival analysis. Rats that had lived for more than 100 days after transplantation were considered to be survivors.

\section{Serum aminotransferases}

Blood specimens from six recipients in each group were collected from tails at 1,3 , $6,24,72 \mathrm{~h}$, and 7 days after surgery. Serum biochemical parameters, including alanine aminotransferase (ALT), aspartate aminotransferase (AST), and total bilirubin (TB), were examined by an automatic chemical analyzer (Hitachi 7600-100, Tokyo, Japan).

\section{Statistical analysis}

Numerical data are reported as means \pm standard deviation. Statistical analysis was performed using SPSS 16.0 for Windows (SPSS, Chicago, IL). The Student $t$-test and the generalized linear model were applied when appropriate. Kaplan-Meier survival analysis was used to evaluate graft survival rate.

\section{RESULTS}

\section{Operative data for DLT and WLT}

Thirty two rats were used as donors and a total of 16 DLTs were performed. WLTs were performed as controls. The operative data are shown in Table 1. The mean operation time 
$(227.71 \pm 22.64$ vs $108.75 \pm 15.53 \mathrm{~min}, \mathrm{P}<0.05)$ and anhepatic time $(23.67 \pm 1.35$ vs $21.13 \pm$ $4.32 \mathrm{~min}, \mathrm{P}<0.05$ ) of DLTs were statistically longer than those of WLTs. The mean ischemia times of the first and second donors for DLTs were $91.73 \pm 13.65$ and $145.07 \pm 18.16 \mathrm{~min}$, respectively.

Table 1. Surgical parameters for each group.

\begin{tabular}{lcc}
\hline Surgical parameter & DLT group $(\mathrm{N}=16)$ & WLT group $(\mathrm{N}=16)$ \\
\hline Total operation time (min) & $227.71 \pm 22.64$ & $108.75 \pm 15.53^{*}$ \\
Ischemia time of primary donor (min) & $91.73 \pm 13.65$ & $58.88 \pm 10.72^{*}$ \\
Ischemia time of secondary donor (min) & $145.07 \pm 18.16$ & NA \\
Anhepatic phase (min) & $25.36 \pm 3.34$ & $21.13 \pm 4.32 *$ \\
Resuscitation time (min) & $75.71 \pm 15.64$ & $70.63 \pm 16.13$ \\
Graft ratio (\%) & $80.57 \pm 9.11$ & $98.15 \pm 4.31^{*}$ \\
\hline
\end{tabular}

$\mathrm{DLT}=$ dual graft liver transplantation; WLT $=$ whole liver transplantation; NA $=$ not applicable. $* \mathrm{P}<0.05$.

\section{DSA evaluation of the hepatic venous and biliary system}

DSA was performed to evaluate the hepatic venous and biliary system following surgery. The results showed that the hepatic PVs, hepatic veins, and biliary tracts of the dual liver grafts were unobstructed, which is critical for survival of the recipients (Figure 2).
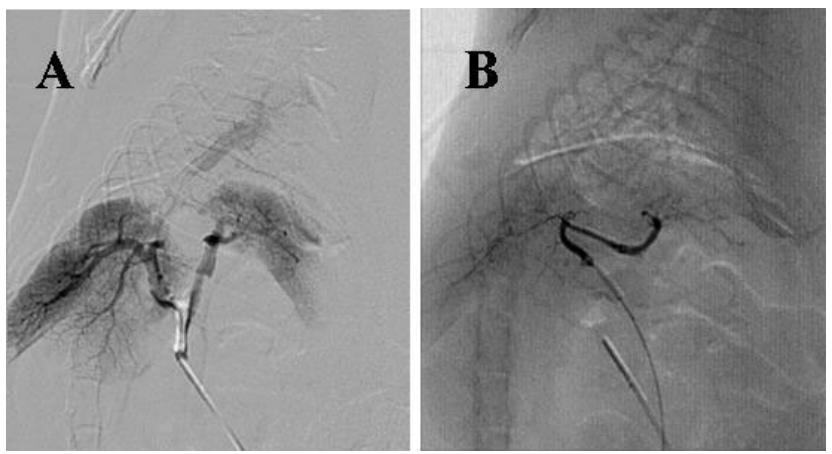

Figure 2. Digital substraction angiography (DSA) evaluation of the hepatic venous and biliary system after dual graft liver transplantation. A. DSA of the hepatic venous system. B. DSA of the biliary tract. The results showed that the hepatic portal veins, hepatic veins, and biliary tracts of the dual liver grafts were unobstructed.

\section{MRI and morphological assessment of liver grafts}

MRI was performed to assess the morphology of dual liver grafts at 7 or 100 days after transplantation. As shown in Figure 3A, on T1-weighted images, both first and second liver grafts showed a normal homogeneous signal without obvious lesions. There was an obvious gap between the dual grafts 7 days after transplantation. However, the gap was replaced by regenerative liver tissue by 100 days after transplantation. Morphologically, both the first and second grafts showed normal structure without obvious necrosis, bleeding, or biliary stasis (Figure 3B). 


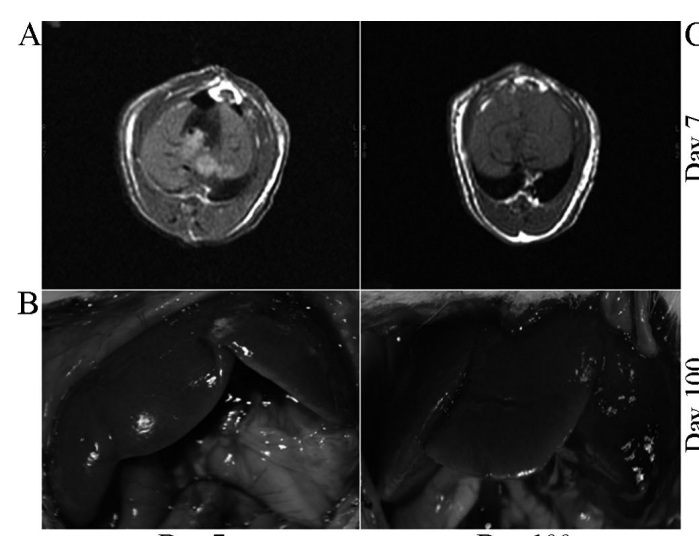

Day 7

Day 100

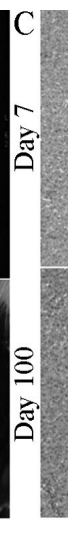

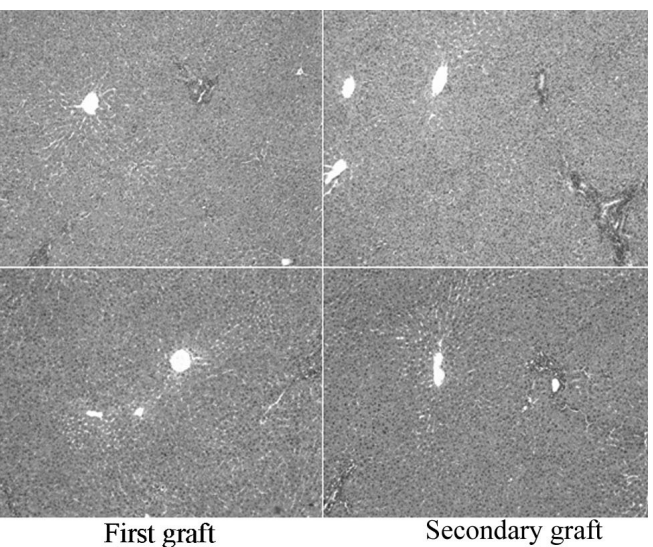

Figure 3. Magnetic resonance imaging (MRI) and pathological assessment of dual liver grafts. A. On MRI T1weighted images, liver grafts showed a normal, homogeneous signal without obvious lesions at 7 and 100 days after transplantation. B. Both the first and second liver grafts showed normal structure without obvious necrosis or bile stasis at 7 and 100 days after transplantation. C. No obvious necrosis, bleeding, or bile stasis was observed in the sinusoidal areas of either the first or second liver graft at 7 and 100 days after transplantation. A small amount of lymphocytic infiltration was observed in the portal zone (100X magnification).

\section{Histological assessment of liver grafts}

The recipient rats were sacrificed 7 or 100 days after transplantation and liver grafts were obtained for further histological examination. As shown in Figure 3C, mild periportal sinusoidal congestion, scattered hepatocellular necrosis and lymphocytic infiltration were observed in both the first and second grafts 7 days after transplantation, which was probably caused by ischemia reperfusion injury. No evidence of cellular destruction, biliary stasis and bleeding were observed in liver grafts 100 days after transplantation.

\section{Biochemical assessment of liver function}

Serum ALT and AST levels were tested after surgery. Serum ALT and AST levels increased at $1 \mathrm{~h}$, reached a peak at $6 \mathrm{~h}$ and then gradually decreased, returning to normal levels $72 \mathrm{~h}$ after DLT or WLT. However, serum total bilirubin levels were continuously elevated throughout the 7 days following liver transplantation. The mean levels of ALT, AST, and TB of the DLT group at 3,6, and $24 \mathrm{~h}$ post-transplantation were significantly higher than those of WLT group. There were no obvious fluctuations in ALT, AST, or TB levels in the sham and normal groups at any time point (Figure 4A).

\section{Long-term survival of recipients with dual grafts}

Ten recipients were used for survival analysis after DLT or WLT. Following DLT, 6 of the 10 recipients survived for more than 100 days. Seven of the 10 recipients survived for more than 100 days after WLT. No significant differences were found in graft survival between the two groups $(\mathrm{P}=0.583$, Figure $4 \mathrm{~B})$. Four of the 10 recipients died within 50 days after DLT: one from abdominal hemorrhage at 3 days; two from PV thrombosis at 11 and 18 days, respectively; and one from biliary obstruction at 50 days post-transplantation. 

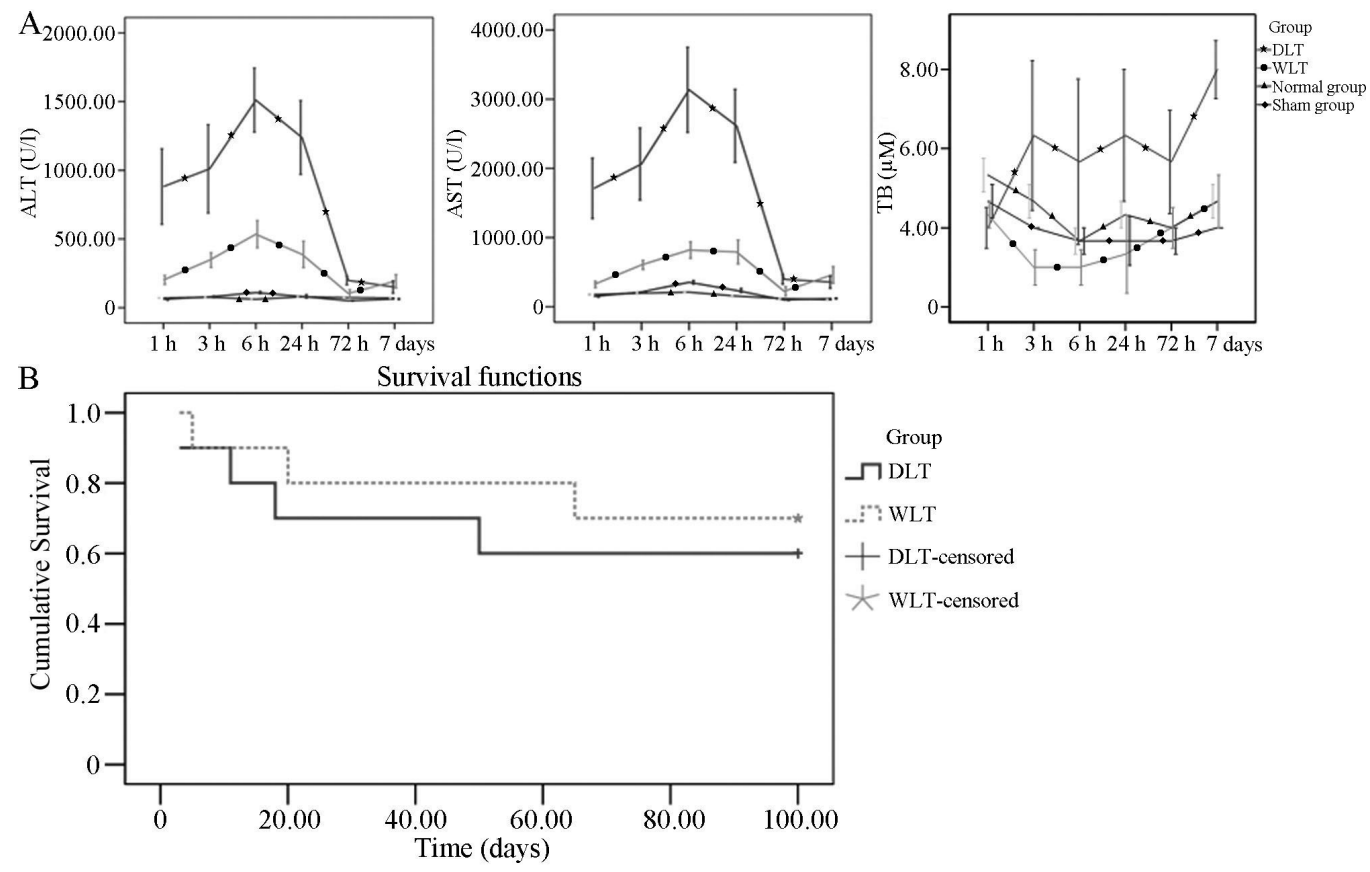

Figure 4. Post-operative recipient graft function and survival analysis. A. Serum alanine aminotransferase, aspartate aminotransferase, and total bilirubin were examined for assessment of graft function. B. No significant differences were found in graft survival between the dual graft liver transplantation and whole liver transplantation groups $(\mathrm{P}=0.583)$.

\section{DISCUSSION}

DLT has been proven to be an effective alternative surgical technique to overcome the potential for SFSS in large recipients, and to ensure donor safety by leaving sufficient remnant liver volume in the donor to facilitate continuing function. However, little is known about the pathophysiological and immune processes after DLT due to the lack of appropriate animal models with long term survival. This study presents an improved technique for establishing a rat model of DLT with long-term survival. The long-term DLT survivors with grafts from two different donors had unobstructed veins and biliary tracts, normal graft function, and wellpreserved hepatic structure.

The key to the success of dual graft liver transplantation lay in clarification of the anatomical relationship of the vessels and bile ducts between the dual liver grafts and the recipient. The inferior vena cava (IVC) passes through the middle lobe of the liver. The opening of the IVC is at the diaphragmatic aspect. Therefore, the middle lobe is required to comprise one of the dual grafts to ensure the integrity of the IVC. It is also worth noting that the second liver graft should be harvested from the right middle lobe rather than from the middle lobe of the second donor's liver. Our previous four operations using the middle lobe as the second liver graft demonstrated that the graft was obviously compressed by the gastric organs, leading to post-transplantation graft dysfunction (Zhang et al., 2012). The middle lobe accounts for $40 \%$ of the total liver, whereas the right middle lobe accounts for only 25 to $30 \%$ (Martins and 
Neuhaus, 2007). Therefore, our previously published model using the middle lobe of the liver as the second donor graft may be not the optimal choice for DLT in rats because the combined graft volume is too large for recipients (Zhang et al., 2012).

The anastomosis of the SHIVC/IHIVC, PVs, and bile ducts between the dual grafts and the recipient was the key factor in determining the outcome after DLT. The SHIVCs of the two liver grafts were sutured into a discoid shape and then manually anastomosed to the SHIVC of the recipient. It is worth noting that orthotopic anastomosis of the SHIVCs should be avoided because this may lead to compression and stenosis of the SHIVCs. We had previously tried to perform orthotopic anastomosis in 3 rats. The rats died at 12, 24, and $45 \mathrm{~h}$, respectively, after the operation as a result of thrombosis in the vena cava caused by vessel compression. Rotation of each dual liver graft through $90^{\circ}$ around the vertical axis successfully avoided the compression of the vena cava. The cuff technique was used to connect the IHIVC of the first liver graft to that of the recipient. The IHIVC of the second liver graft was ligated. Therefore, the venous blood of second graft passed through the SHIVC into systematic circulation.

The two-cuff technique has been successfully used in animal model of WLT, and proven to decrease the time of the anhepatic phase. We used the techniques of the "Y-shaped bridge" and "three-cuff" for the anastomosis of PVs between the dual grafts and the recipient. The confluence point of the left renal vein and the IHIVC is Y-shaped, and thus could be used as the bridging passage. The three ends were anastomosed using a sleeve technique to the PVs of the dual grafts and the PV of the recipient.

Biliary complications have always been considered as the Achilles' heel of liver transplantation, and adversely affect patient and graft survival (Enestvedt et al., 2013). The anastomosis of bile ducts between the dual grafts and the recipient is one of the most important factors in endowing this model of DLT with long-term survival. The "two-stent" technique was adopted for the anastomosis of bile ducts between the dual grafts and the recipient in our study. The CBD of the first graft was anastomosed to the CBD of the recipient with a stent. However, the CBD of the second graft was unable to be directly connected to the CBD of the recipient. To overcome this problem, the $\mathrm{CBD}$ of the second graft was connected to the left hepatic duct of the first graft. This method effectively prevented the potential for bile duct obstruction and leakage.

Ischemia reperfusion injury and anhepatic phase time are also key factors influencing the prognosis of liver transplantation (Henry et al., 2012; Weigand et al., 2012). An anhepatic phase time of not more than $26 \mathrm{~min}$ has been considered to be safe for liver transplantation (Kamada and Calne, 1979), whereas a longer anhepatic phase time has been proven to be negatively associated with graft survival after transplantation (Ijtsma et al., 2009). In our study, the connection between the SHIVC and PV was prepared during the plasty of the donor liver grafts, significantly shortening the time of the anhepatic phase, so that the anhepatic phase time of the DLTs were all within $25 \mathrm{~min}$. Therefore, although the overall operation time and the anhepatic time of DLTs were longer than those of WLTs, resulting in more severe ischemia reperfusion injuries in the DLTs, there was no difference in graft survival between DLT and WLT.

In conclusion, our study provides an improved rat model of dual graft liver transplantation, with long term survival. The main improvements in this model include: i) use of the middle and right middle liver lobes as the dual grafts; ii) use of a "two-stent" technique but not a "Y-shaped prosthesis" for anastomosis of bile ducts between the dual grafts and the recipi- 
ent; and iii) connection of the CBD of the second graft to the left hepatic duct of the first graft. The improved surgical techniques shortened the time of the anhepatic phase, and reduced the incidence of vessel and bile duct obstruction, leading to long-term survival. In the future, we will use different strains of rats as donors and recipients in this model, wherein complicated immune responses may emerge reflective those between human donors and recipients. Thus, we expect our improved rat model of DLT to be utilized as a useful model for evaluating pathophysiological processes involved in liver transplantation, the immune responses between dual grafts and recipients, and the mechanisms underlying liver regeneration in dual grafts following DLT.

\section{ACKNOWLEDGMENTS}

Research supported by grants from the Natural Science Foundation of Zhejiang Province (\#LY13H030002), the Key Science and Technology Innovation Team of Zhejiang Province (\#2009R50038), and the Fundamental Research Funds for the Central Universities (\#2013FZA7009).

\section{REFERENCES}

Chen H, Zhang Y, Han YM, Huguet E, et al. (2013). Dual liver transplantation. J. Zhejiang Univ. Sci. B 14: 178-184.

Chen Z, Zeng Y, Wen TF, Gong S, et al. (2010). Dual grafts live donor liver transplantation for acute-on-chronic hepatitis B liver failure. Transplant. Proc. 42: 4552-4554.

Dahm F, Georgiev P and Clavien PA (2005). Small-for-size syndrome after partial liver transplantation: definition, mechanisms of disease and clinical implications. Am. J. Transplant. 5: 2605-2610.

Enestvedt CK, Malik S, Reese PP, Maskin A, et al. (2013). Biliary complications adversely affect patient and graft survival after liver retransplantation. Liver Transpl. 19: 965-972.

Hashikura Y, Makuuchi M, Kawasaki S, Matsunami H, et al. (1994). Successful living-related partial liver transplantation to an adult patient. Lancet 343: 1233-1234.

Henry SD, Nachber E, Tulipan J, Stone J, et al. (2012). Hypothermic machine preservation reduces molecular markers of ischemia/reperfusion injury in human liver transplantation. Am. J. Transplant. 12: 2477-2486.

Ijtsma AJ, van der Hilst CS, de Boer MT, de Jong KP, et al. (2009). The clinical relevance of the anhepatic phase during liver transplantation. Liver Transpl. 15: 1050-1055.

Kamada N and Calne RY (1979). Orthotopic liver transplantation in the rat. Technique using cuff for portal vein anastomosis and biliary drainage. Transplantation 28: 47-50.

Lee S, Hwang S, Park K, Lee Y, et al. (2001a). An adult-to-adult living donor liver transplant using dual left lobe grafts. Surgery 129: 647-650.

Lee SG, Hwang S, Park KM, Kim KH, et al. (2001b). Seventeen adult-to-adult living donor liver transplantations using dual grafts. Transplant. Proc. 33: 3461-3463.

Martins PN and Neuhaus P (2007). Surgical anatomy of the liver, hepatic vasculature and bile ducts in the rat. Liver Int. 27: 384-392.

Soejima Y, Taketomi A, Ikegami T, Yoshizumi T, et al. (2008). Living donor liver transplantation using dual grafts from two donors: a feasible option to overcome small-for-size graft problems? Am. J. Transplant. 8: 887-892.

Strong RW, Lynch SV, Ong TH, Matsunami H, et al. (1990). Successful liver transplantation from a living donor to her son. N. Engl. J. Med. 322: 1505-1507.

Weigand K, Brost S, Steinebrunner N, Buchler M, et al. (2012). Ischemia/reperfusion injury in liver surgery and transplantation: pathophysiology. HPB Surg. 2012: 176723.

Wertheim JA, Petrowsky H, Saab S, Kupiec-Weglinski JW, et al. (2011). Major challenges limiting liver transplantation in the United States. Am. J. Transplant. 11: 1773-1784.

Yersiz H, Cameron AM, Carmody I, Zimmerman MA, et al. (2006). Split liver transplantation. Transplant. Proc. 38: 602-603.

Zhang Y, He Y, Praseedom RK, Zheng S, et al. (2012). Establishment of animal model of dual liver transplantation in rat. PLoS One 7: e40818. 